\title{
Ueber die Grundlagen der Geometrie
}

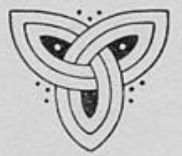 \\ I. Teil. \\ Die geometrischen Axiome im Urteil \\ des Rationalismus und Empirismus \\ von \\ Professor Dr. Christoph Ibrügger.

$$
\stackrel{\nabla}{\nabla}
$$

Beilage zum Programm des Königlichen und Gröning'schen Gymnasiums zu Stargard i. Pom.

1912. Progr.-No. 210.

4. 



\title{
Die geometrischen Axiome im Urteil des Rationalismus und Empirismus.
}

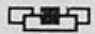

Vorbemerkung. Die folgenden Ausführungen beschäftigen sich mit einem Ausschnitt aus dem Erkenntnisproblem, das sich an die Grundlagen der Geometrie anknüpft. Sie behandeln darauf bezügliche Fragen in historischer Folge, ohne den Anspruch auf Vollständigkeit erheben zu wollen und zu können. - Insbesondere ist damit der Versuch gemacht worden, Schüler der oberen Klassen, die sich für derartige Fragen interessieren, in diese einzuführen. Darum ist Wert darauf gelegt, den Zusammenhang hervorzuheben, in dem das besondere Thema mit dem Erkenntnisproblem überhaupt steht. An eine verbindliche Eingliederung in das mathematische Lehrpensum ist natürlich nicht gedacht; wohl aber kann der gelegentliche Hinweis auf den vorgetragenen Gegenstand - neben der Hervorhebung praktischer Gesichtspunkte, die der mathematische Unterricht ja nicht außer Acht lassen wird, - die Schüler über den Erkenntniswert eines Zweiges der Mathematik orientieren, und damit ist zugleich die Möglichkeit gegeben, sie an der Hand eines bestimmten Beispiels, das der Unterricht darbietet, mit philosophischen Erörterungen bekannt zu machen.
\end{abstract}

1. Dem naiven Denken scheint die Art, wie wir Menschen Erkenntnisse über Tatsachen und Vorgänge in Natur und Geschichte gewinnen, einfach in einer Ab- oder Nachbildung zu bestehen: In ähnlicher Weise, wie in der Camera des Photographen Bilder von außerhalb befindlichen Gegenständen zustande kommen, so spiegele sich in unsrer Seele die Außienwelt. Ohne Bedenken stellt man dem erkennenden Subjekt ein Objekt gegenüber, obwohl es sich im 
Erkenntnisakt lediglich um unsern Bewußtseinsinhalt, unser Wahrnehmen und Denken handelt; und ebenso unbedenklich hält man die vermeintliche Kopie für ein wahrheitsgetreues Abbild, ohne daran zu denken, daß der aufnehmende Apparat - für den man das Erkenntnisvermögen hält infolge seiner physiologischen und psychischen Eigenart zu Änderungen des „Bildes“ Anlaß geben könnte.

Dem eindringenden, philosophischen Denken entstehen aber ernstliche Zweifel, ob die ${ }^{*}$ Auffassung des Erkenntnisvorganges als Abbildung der Wirklichkeit überhaupt haltbar ist, es ergeben sich ihm aus dem Gegensatz von Subjekt und Objekt, Vorstellung und Gegenstand schwer zu lösende, vielleicht unlösbare Probleme, und es wird ihm zu einer wichtigen Aufgabe, festzustellen, welche Bestimmungen in unserm Bewußtseinsinhalt der subjektiven, und welche der objektiven Sphäre angehören.

Ein Gebiet unsers Wissens gibt es, das eine Sonderstellung einzunehmen scheint, auf dem die angegebenen Gegensätze nach vielfach ausgesprochenem Urteil nicht vorhanden sind, die reine Mathematik. Von ihr, im besonderen von der Geometrie - der Wissenschaft vom Raum und den gegenseitigen Beziehungen räumlicher Objekte - heißt es bei E. Cassirer*): „Die Wahrheit der reinen Geometrie besteht nicht darin und sucht nicht darin ihren Beweis, daß in ihren Sätzen irgend welche Verhältnisse der tatsächlichen, konkreten Wirklichkeit zum Ausdruck und zur Nachbildung gelangen. Sie bleibt von der Frage nach dem Sein und Ursprung der Dinge, wie von der nach der Natur und Beschaffenheit unsers Geistes in gleicher Weise unberührt."

Sie hat es mit rom menschlichen Geist selbst geschaffenen Objekten - z. B. der Geraden und dem Kreise - - zu tun, die wenigstens in der Weise, wie und wodurch sie bestimmt sind, in der äußeren Erfahrung nicht angetroffen werden und deshalb nur ideale Existenz haben. Die

*) Das Erkenntnisproblem in der Philosophie und Wissenschaft d. neuer. Zeit. Bd. II, S. 514. 
Leichtigkeit, mit der der Geist über die geometrischen Gebilde verfügt, sie kombiniert und ihre gegenseitigen Beziehungen untersucht, läßt erkennen, daß es sich um seinen eigenen Besitz handelt, bei dem Sein und durch das schaffende Denken Gesetztwerden zusammenfällt, wenn auch eine rein willkürliche Bildung auszuschließen ist. - In logischer Folge schließt sich in der Geometrie Satz an Satz; aber er gilt nur, sofern und weil er in notwendiger deduktiver Verknüpfung aus dem Vorhergehenden hervorgeht. Und diese hypothetische Kette führt schließlich auf gewisse Grundsätze, die als die ersten nicht weiter zurückführbar, nicht beweisbar sind. Die Sicherheit des ganzen Systems beruht also auf unbeweisbaren Sätzen. Ja noch mehr: die Grundgebilde - der Punkt, die Gerade, die Ebene - deren Eigenschaften und Beziehungen die Geometrie untersucht, sind keiner bestimmten Definition fähig, da sie als Individualvorstellungen nicht unter einen Gattungsbegriff subsumiert werden können. Man kann nur den Jedermann geläufigen Vorstellungen einen Namen geben und vielleicht nur negativ angeben, was sie nicht sind. Und endlich die Ausführung der allen andern Konstruktionen zu Grunde liegenden Aufgaben - wie der, eine Gerade zu ziehen oder einen Kreis zu zeichnen - wird postuliert, ohne daß man die Mittel zu ihrer Ausführung angeben könnte, wenn man nicht das rein geometrische Gebiet verlassen will.

Trotzdem bleiben die geometrischen Sätze, als aus zwingenden Schlußfolgerungen entsprungen, apodiktisch gewiß; und vor allem erhebt die Geometrie den Anspruch darauf, kein bloßes Hirngespinst zu sein; sondern was aus den ersten Anfängen durch bloße logische Deduktion, ohne Rücksicht auf die konkrete Wirklichkeit und im voraus, bestimmt wird, gibt bei Anwendung auf dieselbe Wirklichkeit richtige Resultate. Die Geometrie besitzt also objektive Gültigkeit trotz ihrer subjektiven Entstehungsweise und der Unbeweisbarkeit ihrer Grundlagen. Diese so vielfach vollzogene Erprobung wirkt nun rückwärts als Befestigung und Sicherstellung der ersten Bestimmungen, der Axiome. - Aber auch abgesehen hiervon erwecken diese in ihrer klaren Anschaulich- 
keit ein solches Gefühl von Evidenz und Notwendigkeit, daß Zweifel an ihrer Richtigkeit nicht möglich scheinen; und ebensowenig erheben sich Zweifel an ihrer Gültigkeit für die Gegenstände der Wirklichkeit, soweit räumliche Verhältnisse in Frage kommen. So scheinen sie allgemeine und notwendige Normen zu sein, nach denen sich jeder bestimmte räumliche Inhalt richtet. - Und weiter es ist so, als ob das psychologische, anschauende oder denkende, Subjekt bei ihnen nicht in Betracht komme, und daßs G.t und Zeit, wann und wo sie in das Bewußtsein treten, bei ihnen keine Rolle spielen. Dennoch enthalten sie wichtige Beziehungen zum erkennenden Subjekt. Die Geometrie -- wenigstens von ihrer älteren Form, von der wir hier ausgehen, wird dies noch oft behauptet legt die uns allen geläufige Raumanschauung zu Grunde und sucht deren Merkmale zu bestimmen, und da dieselbe in den geometrischen Grundannahmen gewissermaßen in nuce enthalten ist, so geben diese eine Charakteristik unsrer Raumanschauung.

Nach diesem Allen ist es erklärlich, daß sich das Nachdenken oft mit den Grundlagen der Geometrie beschäftigt hat.

2. Nach fünf verschiedenen Richtungen kann man, wenn man sich nicht auf den speziellen Inhalt der Grundsätze beschränkt, die Untersuchung führen. Zunächst in historischer Hinsicht. Wo und in welcher Form treten sie zuerst auf in der Geschichte der mathematischen Wissenschaft, und welche Entwickelung hat letztere durchgemacht, ehe es dem menschlichen Geist gelang, die grundlegenden Sätze in begrifflicher Klarheit herauszuheben? Sodam, wie entwickeln sie sich psychologisch im erkennenden Individuum, und in welchem Verhältnis steht die durch sie ausgeprägte Raumanschauung zu der durch die Sinneswahrnehmungen begründeten? Ein besonderes Interesse nimmt ferner die Erkenntnislehre an den Fragen nach der Natur und dem Ursprung der geometrischen Axiome - und gerade diese Fragen werden in unsern Ausführungen den breitesten Raum einnehmen. Bilden die Axiome einen ursprünglichen Besitz unsers Geistes, werden sie durcl! einen Akt unsers Denkens gesetzt, oder spielt bei 
ihrer Bildung unsre äußere Erfahrung die Hauptrolle, so daß sie die Grundtatsachen der Raumanschauung sind, die uns durch unsre Sinnesempfindungen aufgenötigt werden?*)

Erweitert man sodann den Begriff der Grundlagen nach der methodischen Seite auf die Art, wie die Geometrie bei der Auffindung und Darstellung ihrer Sätze, sowie bei ihren Beweisen verfährt, so eröffnet sich ein neues Feld. Wir sehen daher, wenn wir die Forschungen über die Geschichte der Geometrie außer Acht slassen, daß bei unserm Thema Psychologie und Logik in gleicher Weise interessiert sind. In besonderem Maße erregt es aber endlich das Interesse des Mathematikers, was dadurch bezeugt wird, daß die neueren Untersuchungen über die Grundlagen so der Geometrie, wie der Arithmetik ein eigenes ausgedehntes Gebiet wissenschaftlicher Untersuchung umfassen. $\left.{ }^{* * *}\right)$

3. Von Euklid (er lebte zu Alexandria um 300 v. Chr.) stammt die erste - wenigstens die erste uns überlieferte systematische Darstellung geometrischer Sätze in streng deduktiver Folge. Über sein Leben und seine Persönlichkeit ist sehr wenig bekannt.***) Aber die Arbeit seines Lebens ist

*) Das Ziel dieser Untersuchung ist, um mit den Worten von W. Wundt zu sprechen, „die Grenzen abzustecken, zwischen dem, was unserm Denken gegeben wird, und dem, was es selber hinzubringt, nachzuweisen, bis wohin die logischen Einflüsse innerhalb der Erfahrung berechti?t sind, und von wo sie beginnen, sich ein Recht anzumaßen."

**) Welche Bedeutung diesen Arbeiten beigemessen wird, geht aus den Worten Wellstein's (Encyklopädie d. elemt. Geom. II, S. 3) hervor: "Was die Mathematik zum stolzesten und vollendetsten Vorbild einer reinen Wissenschaft macht, ist nicht sowohl ihre streng deduktive Methode, als vielmehr der Umstand, daß sie in der Lage ist, die Voraussetzungen, auf die sie sich gründet, als "Grundbegriffe“ und "Grundsätze“ an die Spitze ihres Lehrgangs zu stellen und die Tragweite der einzelnen Grundsätze dadurch ins rechte Licht $\mathrm{zu}$ rücken, daß Systeme konstruiert werden, in denen die eine oder die andere dieser Voraussetzungen fehlt." Dem II. Teil unsrer Abhandlung muß es vorbehalten bleiben, ausführlicher hierauf einzugehen.

**) Charakteristisch für ihn ist die stolze Antwort, die er dem König Ptolemaios Soter gab: „In der Geometrie gibt es keinen besonderen Weg für Könige; “ sowie folgende Erzählung: Jemand, der 
erhalten geblieben. Seine Hauptschrift, die Elemente

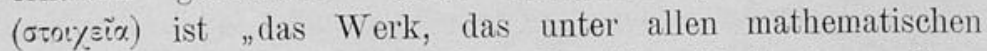
Schriften auf das Geistesleben der Menschheit den weitaus größten Einfluß gehabt hat." Die Elemente beginnen mit Definitionen (öpo $)$ und Axiomen, die er in Forderungssätze

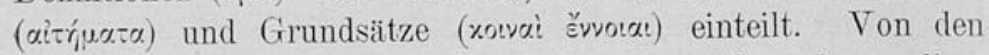
ersten, die Definitionen der Grundbegriffe geben sollen, seien genannt: 1. der Punkt ist das, dessen Teil nichts ist; 2. die Linie aber breitenlose Länge; 4. die Gerade ist die Linie, welche gleichmäßig durch ihre Punkte gesetzt ist; 6. die Ebene ist die Fläche, welche gleichmäßig durch ihre Geraden gesetzt ist; 22. Parallel sind Gerade, welche in derselben Ebene liegen und auf jedem von beiden Teilen ins Unendliche ausgezogen auf keinem von beiden einander treffen. - Die Forderungen, welche die Grundtatsachen, die der Anschauung zu Grunde liegen, enthalten, lauten: Es soll gefordert werden, daß sich 1 . von jedem Punkt bis zu jedem Punkt eine und nur eine Strecke führen lasse, 2. und diese Strecke sich kontinuierlich auf ihrer Geraden ausziehen lasse; 3. um jedes Zentrum sich mit jedem Abstand ein und nur ein Kreis zeichnen lasse; 4. und alle rechten Winkel einander gleich seien; 5. und wenn eine zwei Geraden schneidende Gerade mit ihnen innere an derselben Seite liegende Winkel bildet, die zusammen kleiner sind als zwei rechte, so schneiden sich die beiden geschnittenen Geraden bei unbegrenzter Verlängerung auf der Seite, auf der diese Winkel liegen. - Von den Grundsätzen, den Grundtatsachen der Logik oder auch der Arithmetik, die aber hier auf Raumgrößen bezogen sind, heißen einige: 1. was demselben dritten gleich ist, ist unter sich gleich; 8. und das

angefangen hatte, bei Euklid Geometrie zu treiben, fragte, nachdem er den ersten Satz (der Elemente) gelernt hatte, ,was habe ich nun davon, daß ich das gelernt habe?" Euklid rief seinen Sklaven und sagte: "Gib ihm 3 Obolen, da er lernt um Profit zu machen." (M. Simon, Euclid und die sechs planimetr. Bücher S. 2.) Wir entnehmen dieser Schrift auch für das Folgende mehreres, darunter die Übersetzung der euklidischen Axiome. 
Ganze ist größer als sein Teil; 7. und einander Deckendes ist gleich.

Von diesem euklidischen System der Grundbegriffe und Grundsätze sagt H. Thieme (die Umgestaltung der ElementarGeometrie Progr. Posen 1900): „Im Großen und Ganzen bilden jene Sätze auch heute noch eine geeignete Grundlage für die Geometrie. Mit vollem Recht bewundern wir auch jetzt noch den Scharfblick Euklids, der schon vor mehr denn zwei Jahrtausenden aus den geometrischen Anschauungen diejenigen Elemente auszusondern verstand, die $\mathrm{zu}$ einem folgerichtigen Aufbau des gesamten Gebäudes notwendig und ausreichend sind. Es gilt dies allerdings nur im Grofen und Ganzen. In Grundlage und Aufbau sind doch Mängel vorhanden, welche die Wissenschaft zu einem Neubau veranlaßst haben." - Wir gehen auf den letzten Punkt hier nicht näher ein*), sondern weisen in Bezug auf eine Kritik der Aufstellungen Euklids auf die, auch die Ziele des Schulunterrichts berücksichtigenden Werke hin von Wellstein (s. S. 7), sowie von W. Killing u. H. Hovestadt (Handbuch des math. Unterrichts, Bd. I) und K. Schwering (Handb. d. Elementarmath.); besonder's aber von H. Thieme (d. Elemente d. Geom., II. Teil, I. Bd. der Grundlehren d. Math.), das ein vollständig ausgeführtes System der Geometrie in solcher Begründung enthält, wie es die neuere Wissenschaft fordert.

Wir bemerken hier nur folgendes: Die Elemente Euklids sind vom Standpunkt eines Realismus aus zu verstehen, der die Gegenstände der Außenwelt im Großen und Ganzen so nimmt, wie sie sich der unmittelbaren Wahrnehmung darbieten. - Das ist wohl auch im allgemeinen der Standpunkt der antiken Philosophie. - Die natürliche Raumanschauung

*) Um eine Probe davon zu geben, in welcher Art die Axiome Euklids vervollständigt worden sind, erwähnen wir aus der bedeutenden Schrift von D. Hilbert "die Grundlagen der Geometrie" die Axiome der Anordnung (S. 6), die den Begriff "zwischen “ definieren und auf Grund dieses Begriffs die Anordnung der Punkte auf einer Geraden, in einer Ebene und im Raum ermöglichen. Von diesen Axiomen heißt das dritte: „Unter irgend drei Punkten einer Geraden gibt es stets einen und nur einen, der zwischen den beiden andern liegt." 
bildet ihre Voraussetzung, und darauf beruht, wie unten noch mehr hervortreten wird, die Annahme, die lange geherrscht hat, daß die euklidische Geometrie die einzig mögliche sei. Mit Recht sagt M. Simon: „Euklid hat eine ganze Anzahl stillschweigender Voraussetzungen, ohne die keine anschauliche Geometrie existieren kann. Die Begriffe Dimension, Raum, Richtung werden nicht definiert, ebenso wird die Unendlichkeit des Raumes oder die diese bedingende Unendlichkeit einer Geraden stillschweigend vorausgesetzt." Hierauf ist es auch zurückzuführen, daß die Definitionen der Grundbegriffe, z. B. der Geraden, keine genauen Bestimmungen des Erklärten geben; sie weisen auf etwas hin, was der normalen Anschauung geläufig ist und führen dafür die nötigen Bezeichnungen ein. Begrifflich, in ihren Beziehungen und Verknüpfungen zu den anderen Grundgebilden, werden die Grundbegriffe durch die Forderungssätze definiert, z. B. die Gerade durch die Forderungen 1 und 2, die mit der Detinition 2 zusammenzustellen sind. Bei Euklid ist eben stets die Anschauung hinzuzunehmen.*) „Seine Definitionen

*) Im Gegensatz zu dem Axiomsystem Hilberts. Dieser will die Grundlagen einer allgemeinen euklidischen Geometrie geben, d. h. einer solchen, die für jede drei-dimensionale lineare Mannigfaltigkeit gültig ist (Wellstein), nicht bloß für Punkte, Geraden und Ebenen in der Form, wie sie uns nach ihrem Aussehen erscheinen. Daher sucht er die auf jene Mannigfaltigkeit übertragbaren Eigenschaften begriftlich festzustellen. Er gewinnt sie freilich, wie er sagt, durch logische Analyse aus der räumlichen Anschauung. Daher ist es für ihn so wichtig, alle anschaulichen Nomente, auch diejenigen, die uns selbstverständlich scheinen - s. z. B. das S. 9 angegegebene Axiom - „protokollarisch“ festzulegen. Aber die gewöhnliche euklidische Geometrie ist ihm nur ein spezieller Fall seiner allgemeinen, in der die Begriffe Punkt, Gerade, Ebene gar nicht definiert werden, sondern ihre logische Bestimmung lediglich durch die Axiome erhalten. Nachdem er dann die Axiome gewonnen hat, ist seine Geometrie eine rein logische Beziehungslehre, wenn auch u. E. der Syllogismus nicht das Mittel ist, durch das neue Erkenntnisse gewonnen werden. Euklid will aber immer in unsrer räumlichen Anschauung bleiben, während diese für Hilbert wohl der Ausgangspunkt ist, aber ihm nur ein Paradigma für eine mögliche Geometrie bietet. 
der Begriffe Punkt, Gerade, Ebene, sagt Wellstein (a. a O. S. 111) enthalten ausschlieflich die individuelle, oder wie man es auch auffassen kann, die materielle Seite dieser Begriffe, weshalb denn auch aus diesen Definitionen kein geometrischer Schluß gezogen werden kann." Es wird eben in der Geometrie, wie sie von den griechischen Mathematikern, speziell Euklid, begründet worden ist, die Existenz der Körper in der Außenwelt als selbstverständlich vorausgesetzt, und ihre räumlichen Grundeigenschaften, die sich bei unmittelbarer Wahrnehmung zeigen, werden den Axiomen zu Grunde gelegt. Daher rührt es, daß bei Euklid die physiologischen d. h. die auf das wahrnehmende Subjekt bezüglichen und die begrifflichen $\mathrm{M}_{0}$ mente nicht ganz geschieden werden, und die letzteren nicht vollständig herausgearbeitet worden sind.*)

Noch auf ein Anderes müssen wir in Rücksicht auf spätere Darlegungen kurz eingehen, auf das 5. Postulat oder wie es oft auch heißt das 11. Axiom Euklids - das Parallelenaxiom. Es unterscheidet sich von den übrigen Grundsätzen sehon dadurch, daß das Gefühl der Evidenz, das diese begleitet, bei ihm nicht in gleichem Maße vorhanden ist. Man glaubt eher, es mit einem der Sätze des späteren Lehrgangs zu tun zu haben - wie es denn auch tatsächlich gleichbedeutend ist mit dem Satz, daß die Winkelsumme eines ebenen Dreiecks zwei Rechte beträgt - M. Simon („Zu den Grundlagen der nicht-euklidischen Geometrie" Progr. Straßburg 1891, S. 5) spricht den Grund dafür kurz und treffend dahin aus: „Es ist nicht logisch wie die Grundsätze $1-7$ und nicht anschaulich wie die anderen Forderungssätze, denn

*) Auch das ist noch zu betonen, daß Euklid nicht etwa darauf ausgeht, durch seine Axiome die besondere Form unsrer Raumanschauung $\mathrm{zu}$ charakterisieren und ihre wesentlichen Merkmale aufzustellen. Ihn leitet nur das praktische Interesse, die Grundsätze zusammenzustellen, die zu den Beweisen und Konstruktionen des folgenden Lehrgangs nötig sind. (Vergl. Wundt, Logik 1. Bd., 3. Aufl., S. 481.)

Für den Schulunterricht, für den die wissenschaftliche Fixierung der Raumanschauung Selbstzweck ist, ergibt sich daraus, daß man prinzipiell berechtigt ist, sich auf den Standpunkt Euklids zu stellen. 
das Schneiden kann bei einiger Annäherung an zwei Rechte nicht beobachtet werden, und das Nichtschneiden nie." Sehr alt sind denn auch die Bemühungen der Mathematiker das 5. Postulat - dieses „R reuz" der Mathematik, den „Elecken“, mit dem das große Werk des Euklid behaftet war - dureh ein anschaulicheres Axiom zu ersetzen oder es zu beweisen, d. h. es auf die andern Axiome zurückzuführen. (Vergl. S. 36.)

4. Wir wollen nun, nachdem der Gegenstand, auf den sich unsre Erörterungen beziehen, festgelegt ist, in historischer, — wenngleich richt in vollständiger - Folge besonders die Ansichten, welche über den Ursprung und die Natur der geometrischen Prinzipien geäußert worden sind, vorzuführen suchen, um so das Material für eine spätere systematische Darlegung zu gewinnen, zugleich aber auch um zu zeigen, welche Bedeutung die Prinzipien des geometrischen Wissens für das Erkenntnisproblem haben.

Die Frage nach dem Ursprung menschlicher Erkenntnis - bei der ja zwei Faktoren zu unterscheiden sind, ein empirischer, der aus der Erfahrung stammt, und ein rationaler, der sich auf die Tätigkeit des Denkens bezieht - wird in gegensätzlicher Weise von zwei Hauptrichtungen beantwortet - dem Rationalismus und dem Empirismus. Letzterer sieht in der Erfahrung das wesentliche und bestimmende Moment; das Denken kann nur den durch die Sinnesempfindungen gegebenen Stoff verarbeiten, aber ihm nicht wesentlich Neues hinzufügen. Dem Rationalismus ist das Denken das ausschlaggebende Moment. Nach ihm liefert das Zeugnis der Sinne keine allgemeine und notwendige, und das bedeutet für ihn, keine wissenschaftliche Erkenntnis. Diese wird vielmehr vom Verstande nach immanenten Prinzipien, die ein ursprüngliches Besitztum des Geistes darstellen, liervorgebracht. Das Vorbild des Rationalismus ist die Mathematik.*) In ihr sieht er eine Gewähr für die Möglichkeit seiner Ueberzeugung, da die mathematischen Erkenntnisse aus dem menschlichen Geist gleichsam hervorgesponnen werden; womit dann zusammen-

\#) Kant, Krit. d. rein. Vern. (Ausgabe Reclam S. 548.) 
hängt, daß ihm die Mathematiik und besonders die euklidische Geometrie wegen ihrer streng deduktiven Form vorbildlich erscheint. Indem es endlich zur Erkenntnis der Natur darauf ankommt, die konstanten Beziehungen, die zwischen den Naturkörpern obwalten, nach Maß und Zahl zu ermitteln, d. h. quantitative Bestimmungen enthaltende Naturgesetze aufzustellen, dient die Mathematik dazu, die Natur begreiflich zu machen - ein Moment, das freilich auch der empiristischen Naturforschung durchaus maßgebend ist.

Wir wenden uns zu Plato, dem ersten Vertreter des Rationalismus. Nach ihm wird das bleibend Seiende nur durch begriffliches Denken erkannt, das aus sich heraus in freier Tätigkeit, ohne Vermittelung der Sinne befähigt ist, wahre Erkenntnis, d. h. Erkenntnis des Seienden, zu liefern. In der empirischen Welt, die uns die Sinneswahrnehmung erschließt, mit ihrer Relativität und dem Wechsel der Merkmale und Beschaffenheiten, ist kein gleichbleibendes Sein zu entdecken. Wissen, das von allem Zufall der Wahrnehmung und Meinung unabhängig ist, bietet ihm nur das dialektische und als seine Vorstufe das mathematische Denken. Es ist ihm eine Eigentümlichkeit des Mathematischen, daß es das Bewußtsein zwingt, "sich des Denkens selbst zum Zweck der Wahrheit selbst zu bedienen". "Gerade in der reinen Arithmetik und Geometrie, die sich nicht auf die Dinge der Wahrnehmungswelt, sondern auf die reinen gedanklichen Setzungen der Zahlen und Figuren beziehen, sieht er ein Gebiet von Wahrheit, einen Inbegriff wissenschaftlicher Sätze, die von der Existenz bestimmter empirischer Gegenstände gänzlich unabhängig sind (Cassirer a. a. O. I, S. 37f). Die Anschauungen, die wir von der Geraden, der Kugel u. a. haben, sind ihm nicht Abbilder der Körperwelt, sondern, wenn wir die Wahrnehmungen des Gesichts- und Tastsinns zu einer Geraden oder einer Kugel „zusammenschauen" wollen, so muß das Modell einer derartigen geometrischen Figur uns innerlich bereits vor Augen stehen. Aber erst wenn die flüchtigen Gebilde der „Vorstellung“ zur festen Einheit des Begriffs zusammengeschlossen sind, wird ihnen das "Siegel des Seins" 
aufgedrückt. So bringt die scheinbare und ungenaue Gleichheit der Hölzer und Steine das „Gleiche selbst" ins Bewußtsein. Den Sinn solcher Prädikate zu befestigen, ist Aufgabe der Definition, die damit eine höhere, rein gedankliche Konstanz erschafft, als sie in der Wahrnehmungswelt erreichbar ist. Diesem Standpunkt Platos entspricht es, daf die Geometrie, der bei den Aegyptern der Charakter des Handwerksmäßigen, auf praktische Ziele Abzweckenden anhaftete, ron den griechischen Mathematikern auf die Höhe, exakten begrifflichen Denkens erhoben wurde, und Plato, der Begründer der analytischen Methode und Vorgänger Euklids, ist auch einer der Hauptbegründer der Mathematik als Wissenschaft. Hier an der Quelle der wissenschaftlichen Geometrie ist bereits das Problem über die Bedeutung der empirischen Anschauung und des Denkens erfafit, und dem Denken die Superiorität zugewiesen. Von Plato ist es ferner, wie sich oben zeigte, hervorgehoben, daß die Vorstellungen der elementaren Gebilde, Punkt, Gerade, Ebene, und ihre Beziehungen zu einander nicht lediglich auf einer Ab- und Nachbildung der Wirklichkeit beruhen, sondern auf einer freien Produktivitait des Geistes, der mit schöpferischem Blick die wesentlichen Züge in den Sinneswahrnehmungen erschaut. Das zeigt sich auch in der Ausgestaltung seiner Ideenlehre*), bei der sich Plato vielfach durch Reflexion auf die geometrischen Idealbilder hat leiten lassen. So erscheint die Platonische Idee nie völlig in den Dingen. Ebenso wie die Kante eines wahrgenommenen Körpers nie völlig dem Idealbild der Geraden entspricht. Und wenn er weiter lehrt, daß die Seele durch die Dinge der Körperwelt auf Grund der Aehnlichkeit an die Ideen erinnert werde, so läßt sich dies auf die geometrischen Grundvorstellungen so umdeuten, daß sie keine Wahr-

*) Wie den Sinneswahrnehmungen die Sinneswelt, so entspricht bei Plato den Begriffen die Welt der Ideen, die wahrhaft seiende Wirklichkeit; und wie der Begriff, der das den verschiedenen Wahrnehmungen Gemeinsame enthält, für diese das Bestimmende ist, so ist die Idee das urbildlich Eine der ihr entsprechenden Dinge der Sinnenwelt, und nur sie kann bei unsrer Auffassung der empirischen Dinge vom Denken ergriffen werden. (Rehmke.) 
nehmungsbilder sind, sondern daf ihre Setzung auf Veranlassung der Sinneseindrücke durch spontane Betätigung unsers Geistes geschieht. Gewiß ein richtiger Gedanke, dem man zustimmen muk, wenn man auch der Sinneserfahrung eine wesentliche Rolle zubilligt.

5. Hatte Plato in kühner Konzeption die Welt zu einem Reich an sich seiender Gedanken umgestaltet, so ist der Rationalismus des Begründers der modernen Philosophie Descartes - wesentlich zurückhaltender. Er sucht die begreiflichen, die rationalen Elemente in der uns umgebenden Welt aufzufinden. Dies Rationale findet er in der Mathematik. Rationale Naturerkenntnis und mathematische Physik bedeuten für ihn dasselbe.

Die geometrischen Axiome haben nach Descartes eine dem menschlichen Geiste unmittelbar gegebene Evidenz und eine widerspruclıslose Geltung. „Die mathematischen und geometrischen Ideen" - das Wort ist nicht im platonischen Sinne zu verstehen, es hat die allgemeine Bedeutung von "Vorstellung" - „sind reine Begriffe, in unserm Denken enthalten und als solche aus diesem geschöpft; außser dieser geistigen Wahrheit haben sie auch Uebereinstimmung mit den Dingen draußen und in diesen ihre reelle Stätte"*) Descartes war der Ansicht - die er gegen Hobbes und Gassendi verteidigte - daß die mathematischen Begriffe nicht sowohl Bilder der äußeren Dinge, als gewisse von Natur unserm Geiste angeborene Gemeinbegriffe seien, die vielleicht durch die Dinge, die wir sehen, aus ihrer Latenz hervorgelockt würden, aber doch ihnen gewissermaßen vorausgehen. ${ }^{* *}$ )

Gerade im Hinblick auf die Mathematik hat Descartes wohl zuerst das Prinzip ***) gewonnen, daß jeder in sich klare und deutliche Begriff in seiner Denkbarkeit die Gewähr seiner Gültigkeit besitzt - Quidquid clare ac distincte per-

*) Baumann, die Lehren von Raum, Zeit und Mathematik. Bd. I, S. 87 .

**) Baumann, S. 83 .

***) Auf den Ausgangspunkt seiner Metaphysik „cogito ergo sum" und seine Lehre von Gott soll hier nicht eingegangen werden. 
cipio, verum est. Da Denknotwendigkeit und Realität dem älteren Rationalismus Wechselbegriffe sind - eine Annahme, welche erst von Kant endgültig widerlegt wurde - ist es Descartes nicht zweifelhaft, daß die mathematischen Grundsätze und die aus ihnen abgeleiteten Sätze trotz ihrer mentalen Existenz für die Wirklichkeit gelten, und daß durch ihre Anwendung objektive Erkenntnis gewonnen wird. Von der Körperwelt kann nur soviel erkannt werden, als deutlich vorgestellt werden kann, und das ist das Mathematische. Intuition und Deduktion - wie sie in der Geometrie zur Anwendung kommen — sind die fundamentalen Erkenntnismittel unsers Geistes. Die räumliche Ausdehnung des physikalischen Körpers ist seine einzige erkennbare Bestimmtheit. Corpus est res extensa. - Die Bedeutung der Sinneswahrnehmung ist für unsere Erkenntnis im allgemeinen dieselbe wie in der Geometrie: sie kann den ersten Anstoß zur Bildung von Begriffen und Sätzen geben. Aber erst mit der Demonstration haben wir eigentliche, vollendete Wissenschaft. Die auf empirischem Wege gewonnene Kunde beruht auf der „Einbildung“ (imaginatio), diejenige des mathematisch Bestimmten auf "Verstandeserkenntnis" (intellectio), und so sehr Descartes die Hülfe zu schätzen weiß, welche die Erfahrung für Erforschung des Qualitativen gewährt, so beruht ihm wissenschaftliche Einsicht auf der letzteren.*) (Windelband, Gesch. d. Philos., S. 322.)

*) Auch in der Mathematik unterscheidet er diese beiden Erkenntnisarten: das Dreieck mit seinen 3 Seiten sich vorstellen, heißt es sich einbilden, das Chiliogon, das Tausendeck, aber denken heißt, es ohne Einbildungskraft einsehen. Bei Letzterem möge unsre Raumphantasie versagen, aber es werde vieles deutlich und klar von ihm bewiesen, und das Ganze werde gleichzeitig klar eingesehen, obschon wir uns das Ganze nicht gleichzeitig ,einbilden“ könnten. - Die Intellectio ist Descartes daher die mathematische Erkenntniskraft. - Er weist ferner darauf hin, daß die Geometrie, indem sie sich der Instrumente Lineal und Zirkel bedient, beim wirklichen Zeichnen der Figuren in das Gebiet der Mechanik übergreift. Das eigentlich Mathematische sei aber die Genauigkeit des urteilenden und schließenden Denkens. So stellt auch Descartes das logische Moment in der Geometrie höher als das anschauliche - wie es nicht anders 
6. In noch weiter gehender Weise ist Spinoza's Rationalismus an der Mathematik orientiert. Der Zusammenhang ihrer Wahrheiten, in welchem niemals von Zweck, Wirksamkeit, Tätigkeit, sondern nur von konsequenten Folgen der einen aus der andern die Rede ist, schien ihm das Ideal der Auffassung zu sein, nach dessen Muster alle Zusammenhänge in der Welt erklärt werden müßten. Nur das sei begreiflich, was der Verstand selbst erschafft. Darum ist ihm die Geometrie, die ihre Gebilde durch ihre Definitionen und Konstruktionen selbst hervorbringt, das Vorbild alles wahren Erkennens und die einzige und unbedingte Form alles Folgerns und Schließens. Denn nach seiner religiösen Grundüberzeugung gehen alle Dinge aus dem einheitlichen Wesen Gottes hervor, und nach seinem Grundsatz: ordo et connexio rerum idem est ac ordo et connexio idearum ist dieses Hervorgehen als ein logisches Hervorgehen aufzufassen.*)

Somit ist es für Spinoza keine leere Form, wenn er z. B. seine Ethik more geometrico behandelt, d. h. wenn er

*) "So gewinnt die Mathematik eine andere und weitergreifende Bedeutung als ihr früher bei Descartes und Galilei zukam. Wenn Descartes alle physikalische Wirklichkeit in rein geometrische Beziehungen auflöst, so versäumt er es doch nicht hervorzuheben, daß es sich ihm hierbei nicht um eine Ordnung des Seins, sondern um die Ordnung des Erkennens handelt, daß nicht die innere Wesenheit der Sachen, sondern die logische Stellung der Begriffe es ist, die er im Auge hat ... Für Spinoza gibt es keine derartige Beschränkung; die wahrhafte Verknüpfung der Begriffe trägt die Gewähr der absoluten Realität der Objekte unmittelbar in sich. Die strenge, durch sich selber bestimmte Folge der Gedanken, die Art, wie der eine aus dem andern deduktiv erwächst, spiegelt den realen Prozeß wieder, kraft dessen die Finzeldinge ins Dasein treten." (Cassirer a. a. O. Bd. II, S. 17.)

zu erwarten ist bei dem Begründer der analytischen Geometrie, der es verstanden hat, die in der Anschauung gegebenen Beziehungen der Raumgebilde durch die Rechnung auszudrücken, d, h. sie von der steten Rücksichtnahme auf die Anschauung frei zu machen und sie daher einer logischen Behandlung zu unterwerfen. 
nach Aufstellung von Definitionen und Axiomen seine Darlegung in die Form einzelner Lehrsätze (Propositionen) hineinprelst - unter Angabe von Foraussetzung und Behauptung und sie genau in der Weise, wie Euklid verfährt, streng "beweist". Diese dem Euklidischen Beweisgang nachgebildete, geometrische Methode fand vielfache Nachahmung. Sie verkennt, daß eine wesentliche Eigenschaft der Geometrie die Anschaulichkeit ist. Hierauf beruht die Evidenz ihrer Axiome, die sich keineswegs - wie eine Betrachtung z. B. der "Axiome" in Spinoza's Ethik zeigt - auf Gebiete übertragen läßt, denen die Anschaulichkeit fehlt.*)

7. Hatte der Rationalismus versucht, die Wirklichkeit mit Hülfe logischer Formen zu begreifen, schien ihm die Mathematik als Ideal der Wissenschaft, so treten ihm als die Hauptvertreter des Empirismus die Engländer Locke und Hume entgegen. Die Frage nach dem psychologischen Ursprung unsers Wissens tritt in den Vordergrund und mit ihr die Hervorhebung der Sinnesempfindung als Erkenntnisquelle.

Alle unsere Erkenntnis entspringt aus sinnlichen Empfindungen, die selbst wieder - entsprechend der Auffassung des Realismus - auf Eindrïcke äuferer Dinge zurückgeführt werden. Das ist der Ausgangspunkt der Locke'schen Lehren. Unsre Eifahrung beruht nicht so sehr auf produktiver Tätigkeit unser's Verstandes, als vielmehr auf einer passiven Aufnahme von Sinneseindräken, denen gegenüber die Seele sich wie ein leeres Blatt verhält - so daß wir von „angeborenen Ideen “ nichts in ihr antreffen. Weitere Ueberlegung lehrt uns dann, die sinnlichen Qualitäten, Farbe, Geruch, Ton etc., als Beschaffenheiten, die den Dingen an sich nicht zukommen, von den mathematischen Eigenschaften zu unterscheiden, die im Gegensatz zu jenen sekundären, subjektiven Qualitäten eigene Prädikate der

- Eine wuchtige Widerlegung findet die geometrische Methode in Kant's Kritik d. rein. Vernunft (Ausgabe von Reclam, S. $548 \mathrm{ff}$ ), wo er ausführt: „Die philosophische Erkenntnis ist die Vernunfterkenntnis aus Begriffen, die mathematische aus der Konstruktion der Begriffe." 
Dinge sind, nämlich Ausdehnung, Größe, Gestalt und Bewegurg. - Von seinen Ansichten über Mathematik sei nur einiges hervorgehoben. Die Grundprinzipien der Geometrie werden durch intuitive Erkenntnis gewonnen, und diese anschauliche Erkenntnis, mit der ich z. B. wahrnehme, daß ein Bogen eines Kreises kleiner ist als der ganze Kreis, ist die höchste Gewißheit menschlichen Erkennens. Die geometrischen Beweise erfolgen auf Grund von Anschauung, freilich nicht durch unmittelbare wie bei den Grunderkenntnissen, sondern durch nach und nach zustande gebrachte. So erkennt man z. B., daß die Winkelsumme eines Dreiecks zwei Rechte beträgt, wenn man die Winkel an einer Ecke des Dreiecks in passender Weise zusammenfügt. Weil daher die Anschauung das Wesentliche in der Mathematik ist, darum haben die Axiome der allgemeinen Größenlehre und die Syllogismen wohl Wert für eine kurze und scharfe Lehrmethode, sie sind aber nicht die Grundlagen und Mittel des Erkennens, sofern es erfindend ist, auch helfen sie einem nichts dazu, einen Beweis einzusehen. - Dem gegenüber sei darauf hingewiesen, welche Rolle Leibniz der Anschauung zuerteilt. Nach ihm ist die Kraft des Beweises unabhängig von der gezeichneten Figur, die vielmehr nur als Anhalt dient und das Verständnis erleichtert. Die Axiome und die Definitionen der Begriffe, sowie die schon bewiesenen Sätze sind die Grundlagen des Beweises. Auf "Imagination" — die nur eine verworrene Einsicht liefert -- darf eine Wissenschaft nicht gegründet werden. Somit hat auch die Geometrie für ihn einen wesentlich logischen Charakter: Bei Kant, der den Begriff der reinen Anschauung begründete, werden wir diesen Gegensätzen, die auch in der jetzigen Zeit noch viel umstritten sind, wieder begegnen. - Da für Locke nicht der Allgemeinbegriff, sondern die bestimmte empirische Einzelvorstellung maßgebend ist - z. B. eines Dreiecks mit bestimmten Seiten und Winkelverhältnissen, wie sie uns eben eine rein psychologische Betrachtung nur bieten kann - so ist es ihm nicht gelungen, die allgemeine Gültigkeit der geometrischen Sätze zu erklären, insofern diese nicht nur für ein vorliegendes, bestimmtes 
Dreieck, sondern für alle Dreiecke zu gelten beanspruchen.*) Diese Schwierigkeit haftet, worauf auch Leibniz bereits hingewiesen hat, jeder rein sensualistischen Erkenntnislehre an. Nach ihr steht eben die Welt der äußeren Dinge derjenigen unsrer Ideen unvermittelt und getrennt gegenüber. Bleibt es einmal schon ein Rätsel, wie unser Denken es anfängt, sich der äußeren Gegenstände zu bemächtigen, so ist es andererseits auch ungewiß, wie man bei dieser Abbildungslehre mehr aussagen kann, als was sich nur auf den abgebildeten einzelnen Fall bezieht, so daß die Aufstellung allgemeiner Gesetze unmöglich wird. Und endlich bleibt ihr auch die Annahme, daß die Ergebnisse unsers Denkens für die Wirklichkeit Geltung haben, daß Denken und Sein sich entsprechen, unerklärlich.

Das zeigt sich auch bei dem letzten Punkt, den wir bei Locke erwähnen wollen. Wie es vor ihm schon vielfach ausgesprochen war, lehrte auch er, daß die geometrischen Sätze zunächst nur für die Ideen, d. h. für die Vorstellungen in unserm Geiste ${ }^{* *}$ ) richtig sind. Die Eigenschaften eines Kreises oder eines Rechtecks bezögen sich zunächst nur auf Figuren, die diesen Ideen entsprächen. Aber die geometrischen Sätze hätten neben ihrer idealen Geltung auch Bedeutung für die Wirklichkeit der Dinge, sofern diese und nur insoweit diese mit den geometrischen Mustern im Geiste übereinstimmten, d. h. soweit die angenommenen Voraussetzungen in der Wirklichkeit zuträfen. Wir fügen hinzu, daß aber die Ueberzeugung zu ergänzen ist, daß denknotwendige Folgen in uns mit naturnotwendigen Folgen außer uns übereinstimmen müssen. Diese Ueberzeugung von der objektiven Gültigkeit unsers

*) Bei dieser Gelegenheit sei darauf hingewiesen, wie viel einem Quartaner zugemutet wird, wenn der Lehrer irgend ein Dreieck an die Tafel zeichnet, an diesem nun gewisse Eigenschaften deduziert, und dann ohne Weiteres den Schüler lernen läßt: in jedem Dreieck sind die und die Eigenschaften vorhanden.

**) Die Annahme solcher Vorstellungen, die in der Wirklichkeit nichts Entsprechendes haben, ist vom Locke'schen Standpunkt aus eine Inkonsequenz. 
Denkens ist es, die über eine rein sensualistische Theorie hinausführt.

8. Eine konsequente Durchführung des Empirismus finden wir bei dem scharfsinnigen Schotten Hume, dessen Lehre von Windelband (Gesch. d. Phil.) so charakterisiert wird: „Es gibt keine Erkenntnis dessen, was die Dinge sind und wie sie wirken; wir können nur sagen, was wir empfinden, welche räumliche und zeitliche Anordnung und welche Aehnlichkeitsverhältnisse wir zwischen ihnen erleben." Was zu unsern Sinneseindrücken den Anlaß gibt, läßt er dahingestellt. Aber, was den Inhalt und die Betätigung unsers Geistes betrifft, so gibt es nach ihm nur Impressionen*) und Ideen, von denen die letzteren die Kopien der ersteren sind. Auch nach ihm haben alle allgemeinen und abstrakten Ideen nur Berechtigung in solcher Abbildung und einer Zusammenfassung von bestimmten Einzelvorstellungen, welche letzteren nur an einen allgemeinen Namen angeschlossen werden. Es gibt zwei Arten von Erkenntnisobjekten: Relationen von Ideen und Tatsachen -- das Gebiet des formal-begrifflichen Wissens, dessen Muster die Mathematik ist, und dasjenige des empirischen, des Tatsachenwissens. Sätze von der Art des pythagoreischen Lehrsatzes können durch bloße Tätigkeit des Denkens entdeckt werden, ohne Abhängigkeit von dem, was irgendwo in der Welt existiert. Wenngleich es niemals einen Kreis oder ein Dreieck in der Natur gäbe, so würden die Wahrheiten, die von Euklid bewiesen sind, für immer ihre Gewißheit und Evidenz behalten. Denn wenn die Voraussetzungen, die Euklid macht, gelten, so sind die von ihm bewiesenen Sätze denknotwendig; ihr Gegenteil einzusehen, ist nicht möglich. Auf diesem Gebiet gibt es beweisbares Wissen, dem das Merkmal der Allgemeinheit und Notwendigkeit zukommt. Will man nur diese Eikenntnis als echte Wissenschaft gelten lassen, so ist allerdings die reine Mathematik eine W issenschaft, aber anch nur, so lange sie sich innerhalb der Begriffswelt bewegt. Sowie sie auf Tatsachen der Natur-

*) Für unsern Gegenstand kommen als Impressionen nur die Sinneseindrücke in Betracht. 
forschung angewandt wird, hört die Apodiktizität auf, da Tatsachen nicht apodiktisch gewiß sind. Denn das Gegenteil jeder empirischen Tatsache - etwa daß morgen die Sonne nicht wieder aufgehen wird - ist immer möglich und kann mit derselben Leichtigkeit und Deutlichkeit vorgestellt werden, als wenn es der Wirklichkeit gemäß wäre. Auf dem Gebiet des empirischen Wissens gibt es nur relative und wahrscheinliche Gewißheit, also keine Wissenschaft im alten Sinne.*)

Hume's Ansichten über Mathematik im einzelnen sind besonders in seiner Schrift "Treatise on human nature" enthalten, für die ganz einzutreten er in ihrer Umarbeitung (Inquiry concerning human understanding) ablehnt. Aber sie stimmen mit seiner Grundüberzeugung, daß jede Idee die Kopie einer Impression ist, überein, so daß wir sie trotz jenes Einspruchs nicht ganz übergehen können. Auch sind sie geeignet zu zeigen, daß der bloße Sensualismus eine genaue Geometrie nicht begründen kann. Dieser kann nicht anders, er muß die geometrischen Grundgebilde irgendwie substanzialisieren, damit sie überhaupt wahrgenommen werden können. Ein ausdehnungsloser Punkt, eine Linie als Länge ohne Breite, eine Ebene sind Forderungen, mit denen wir uns über die Ungenauigkeit der Wahrnehmung erheben (F. Klein). In den Impressionen sind sie aber nirgends gegeben und darum sind diese Idealbegriffe nach Hume sogar bloße Fiktionen, die ebenso nutzlos als unverständlich sind. Ferner macht die unbeschränkte Teilbarkeit einer Raumstrecke seiner Theorie Schwierigkeiten; - selbstverständlich, weil, wenn die Streckenelemente unter die Grenze der Wahrnehmbarkeit

*) „Hume hob kräftigst hervor, daß die mathematische Erkenntnis vor jener empirischen darin etwas voraus habe, sla wir be ${ }_{i}$ den "Tatsachen" immer etwas letztlich einfach hinnehmen müssen, ohne es von Grund auf verstehen zu können. Ja, in der endgültigen Zerstörung aller Hoffnungen, als sei ein solcher empirischer Rest restlos in reines Denken aufzulösen, besteht das größte und unver. gänglichste aller Verdienste Hume's um die Erkenntnistheorie und durch sie auch um die Erkenntnispraxis der empirischen Forschung." (A. Höfler, Didaktik d. math. Unterr., S. 450.) 
hinabsinken, ihre Unterscheidbarkeit aufhört.*) Endlich, um noch ein bei Hume vorkommendes bestimmtes Beispiel anzuführen, kann der Sinneseindruck bei zwei sich schneidenden Geraden - zumal wenn ihr Winkel klein ist - nicht entscheiden, ob sie nicht statt eines Punktes ein Linienstück gemeinsam haben. So kommt nach Hume in die ganze Geometrie ein unsicheres Element, und es ergibt sich ihm, daß diese auf Ideen anfgebaut ist, die nicht genau und auf Grundsätzen, die nicht völlig wahr sind. Einen Ausweg aus diesen Schwierigkeiten sieht er darin, daß auch die Mathematik den Anspruch einer unbedingten Allgemeinheit des Urteils aufgibt und das induktive Verfahren der Naturwissenschaft nachahmt. So finden wir bei Hume bereits Ansichten, die in der späteren Zeit von Stuart Mill und Helmholtz und in anderer Begründung noch von manchen anderen entwickelt sind, daß nämlich die Mathematik unter die Naturwissenschaften $\mathrm{zu}$ stellen ist. $\left.{ }^{* *}\right)$ Andererseits gibt Hume bereits dem Gedanken Ausdruck, man könne die Bildung einer Geometrie versuchen, die sich lediglich im Kreise des Sinnlich-wahrnehmbaren zu halten habe - kurz die Ausführung der "natürlichen Geometrie". (S. Wellstein und Killing-Hovestadt.)

Aus der Zeit Hume's erwähnen wir noch eine Aeußerung d'Alemberts (Eléments de philosophie 1759), dessen sensualistische Kritik gerade vor den mathematischen Grundlagen Halt macht. Nach ihm verkörpern die geometrischen Begriffe sich niemals in irgend einer einzigen Impression, noch sind sie an ihr zu messen. So gewiß wir, um zu ihnen zu gelangen, von sinnlichen Eindrücken den Anfang machen müßten, so wenig vermöchten diese allein ihren Gehalt und ihre Bildung zu erklären,

*) Wohingegen darauf hinzuweisen ist, daß ein bestimmter Raumteil eben kein körperlich abgegrenztes Stück ist, daß wir daher in ihm beliebig viele Punkte als Ausgänge beliebig angenommener Erstreckungen setzen dürfen. Freilich ist auch die Setzung solcher Punkte ein Postutat.

*) S. S. 40. 
und er macht auf den eigentümlichen Abstraktions- und Grenzprozeß aufmerksam, der zu ihrer Bildung führt.*)

9. Schon Leibriz hatte sich gegen den Sensualismus Locke's gewandt, indem er zwar dessen Satz zustimmte ,.nihil est in intellectu, quod non prius fuerit in sensibus", jedocl. hinzufügte: ,nisi intellectus ipse". A ber besonder's Kant ist es gewesen, der einen Ausgleich rationalistischer und empiristischer Lehren begründet hat. Hatte der Rationalismus geglaubt, durch reines Denken absolute Erkenntnis gewinnen zu können, die durch die Sinne nicht möglich sei, während dem Empirismus nur die Wahrnehmung Erkenntnis vermittelte, die dadurch aber ihres absoluten Charakters entkleidet würde, so lehrte Kant: es gibt Erkeuntnis a priori d. h. aus reiner Vernunft und unabhängig von der Erfahrung, aber nur von den Dingen, wie sie uns erscheinen, nicht wie sie an sich sind; und insofern den Stoff für die Erscheinungen nur die Wahrnehmung liefert, während die Synthesis der Vernunfttätigkeit diesen Stoff formt und das Mannigfaltige zu einer Einheit verbindet, kann wissenschaftliche Erkenntnis nicht über die Sphäre der sinnlichen Anschauung hinausgehen. Unsere Erfahrung - hier in dem Sinne als Vorstellung eines Zusammenhangs der Dinge nach allgemeinen Gesetzen hebt mit der Wahrnehmung an, stammt aber nicht ganz aus dieser. Den Rohstoff für unsre Erkenntnis geben uns die Empfindungen, aber er wird von uns in spontaner Tätigkeit unter der Form räumlich-zeitlicher Anschauung aufgenommen und von unserm Verstand nach ilım eigentümlichen Gesetzen zur Erkenntnis geformt. Beides, die Verknüpfung der Empfindungen zu Anschauungen in den Formen von Raum und Zeit, sowie die Verknüpfung der Anschauungen nach den Begriffen von Substanz, Ursache und Wirkung u. a. ist die Bedingung für das Zustandekommen objektiv gültiger und gesetzmäßig geordneter d. h. wissenschaftlicher Erkenntnisse. Also nur, wenn das Denken sich mit dem anschaulich Gegebenen befaßst, durch die Verbindung von Anschauung und

, Zu obigen Ausführungen vergl. die betr. Abschnitte in Baumann und Cassirer. 
Begriff — denen die Frkenntnisvermögen Sinnlichkeit und Verstand entsprechen - werden wir zu Erkenntnissen geführt. Denken ist nicht gleich erkennen. Aber aus der Sinneswahrnehmung stammt nur der Stoff, nicht aber die Form unsrer Erkenntnis. Die Form ist eine Zutat unsrer Auffassung. Sie ist a priori, unabhängig von der Erfahrung, während jener aus der Sinneswahrnehmung stammende Anteil, a posteriori genannt wird. - Woran erkennen wir nun in unsrer Erfahrung die in allem Mannigfaltigen sich gleichartig bleibenden, a priorischen Elemente? Sie zeichnen sich durch den Charakter der Allgemeinheit und Notwendigkeit aus. Bei allem Wissen, das aus der Sinneserfahrung stammt, - hierin stimmt Kant mit Hume überein - kann nur gesagt werden, dies und das hat für mich als empfindendes Subjekt tatsächlich Geltung. Bedeutet es schon einen Sprung, wenn ich meiner subjektiven Sinneserfahrung objektive Gültigkeit zuschreibe, so fehlt mir bei ilır ganz die Ueberzeugung, daß sie denknotwendig eintreten mußte. Deshalb folgert Kant, soweit der Charakter der Allgemeinheit und Notwendigkeit unsre Erkenntnis begleitet, kann diese nicht aus der Erfalıung stammen; es bleibt also für sie als Quelle nur der zweite Faktor, der unsre Erkenntnis beeinflußt, übrig - nämlich das erkennende Bewußtsein. Es ist ihm aber nicht zweifelhaft, daf es apriorische Urteile gibt, sowohl in der Wissenschaft als auch in dem Bewußtsein des täglichen Lebens. Besonders findet er sie in den Sätzen der Mathematik. Ihnen haftet das Merkmal an, daß sie „Notwendigkeit bei sich fülıren, welche aus Erfahrung nicht abgenommen werden kann."

Das Vorhandensein der Mathematik als Wissenschaft - und hiermit kehren wir wieder zu unserem Thema zurück - bietet ihm also die Gewähr dafür, daß es in unsrer Erkenntnis apriorische Bestandteile gibt. Andererseits stellt er die bekannte Frage, wie ist Mathematik als Wissenschaft möglich? Wir geben ihr eine etwas andere Wendung und suchen im Kant'schen Sinne vier Fragen zu beantworten. 1. Was ist der Grund für die Apodiktizität der geometrischen Sätze? 2. Welches ist die besondere Erkenntnisart, aus der 
die geometrischen Wahrheiten geschöpft werden, oder welcher Art ist die Betätigung des Verstandes bei ihrer Schöpfung? 3. Wie erklärt sich das Evidenzgefühl, das wir bei ihren Axiomen haben? 4. Warum sind ihre Sätze keine Hirngespinste, d. h. warum haben sie für die Wirklichkeit objektive Gültigkeit?

Die Antwort auf die erste Frage lautet so, wie sie auch früher schon gegeben ist: Die Mathematik ist ein Erkenntnis aus reinem Verstande und reiner Vernunft; es handelt sich bei ihr um Objekte, die nicht aus der Erfahrung stammen. sondern um solche, die der Verstand selbst hervorgebracht hat. Darum kann er a priori über sie urteilen. A ber Kant bestimmt das der Geometrie eigentümliche Verfahren genauer, als es vor ihm geschehen ist, und vor allem findet er eine alle Fragen umfassende Antwort in der Apriorität des Raumes.

Wir gehen zunächst auf die zweite Frage ein: Kant unterscheidet synthetische und analytische Urteile. Die ersteren sind solche, die unsere Erkenntnis erweitern, indem durch sie im Prädikat neue Bestimmungen zum Subjekt hinzugefügt werden, die nicht im Begriff des Subjekts enthalten sind, während die analytischen Urteile $\log$ ische Folgerungen sind, d. h. nur die Merkmale deutlich zum Ausdruck bringen, die zum Inhalt des Subjektbegriffes gehören. So ist das Urteil "alle Körper sind ausgedehnt" ein analytisches, weil die Ausdehnung zum Begriff des Körpers gehört. Dagegen "Einige" (so weit sie uns bekannt sind) "Körper sind schwer" ist ein synthetisches Urteil, weil wir von der Schwere der Körper nur durch die Erfahrung - die also zu dem Begriffe des Körpers etwas Neues hinzufügt - Kenntnis gewinnen. „Gott ist das höchste Wesen" ist ein analytisches Urteil; dagegen "es gibt einen Gott" - ein Urteil also, das eine Existenz behauptet, ist, wie alle Existenzialurteile, synthetisch. - Daß durch Erfahrung, durch Wahrnehmung, etwa in den Naturwissenschaften, synthetische Urteile gewonnen werden können, ist an sich klar. Denn hier ist es eben die Erfahrung, die Neues kennen lehrt, z. B. daß Natriumsalze die BunsenFlamme gelb färben. Welches ist aber die erweiternde Er- 
kenntnisquelle in der Geometrie, in deren Urteilen wir es trotz ihrer Unabhängigkeit von der Erfahrung zweifellos mit Erweiterungsurteilen zu tun haben? Das ist die Anschauung, sagt Kant - eine Antwort, die wir erklärlich finden, wenn wir uns an die Grundlagen der euklidischen Geometrie (S. 9) -. und diese kam für Kant nur in Eetracht - erinnern. Insbesondere sind nach seiner Meinung die Grundsätze der Geometrie synthetische Urteile. Als Beispiel führt er an: „daß die gerade Linie zwischen 2 Punkten die kürzeste sei, ist ein synthetischer Satz. Denn mein Begriff von der Geraden enthält nichts von Größe, sondern nur eine Qualität. Der Begriff des Kürzesten kommt also gänzlich hinzu und kann durch keine Zergliederung aus dem Begriff der geraden Linie gezogen werden. Anschauung muß also hier zu Hilfe genommen werden, vermittelst deren allein die Synthesis möglich ist."

Gemeint ist von Kant nicht die empirische Ansch sung, wie sie uns in dem Wahrnehmungsinhalt der einzelnen Sinne entgegentritt, sondern die Möglichkeit, daß ihre Begriffe in rein gedanklicher Konstruktion - durch produktive Einbildungskraft - anschaulich dargestellt werden können, ist es, die den besondern Charakter der Mathematik, speziell der Geometrie, bedingt. Um dies noch näher darzulegen, gehen wir von dem oft gehörten Satze - z. B. in der Pädagogik - aus, der Vorzug der Geometrie beruhe auf ihrer Anschaulichkeit, womit man meistens die Möglichkeit bezeichnen will, daß man ihre Objekte durch Zeichnungen oder Modelle darstellen kann. Die Anschauungen, die man so erhält, sind aber empirische; und wäre diese empirische Anschaulichkeit der Hauptzug der Geometrie, so würde sie auf Sinneswahrnehmung beruhen, und ihre durch Anschauen empirischer Objekte gewonnenen Sätze würden dann der Notwendigkeit entbehren. „Es würde nur heißen: man bemerkt es jederzeit so, und der Satz gilt nur so weit, als unsre Wahrnehmung bis dahin sich erstreckt hat." (Proleg. Ausg. Reclam S. 62.) ' Auch die Konstruktion vermittelst der Raumphantasie, in der Vorstellung, meint Kant mit der reinen Anschauung nicht. Diese Vorstellungen sind 
sogar, wie F. Klein hervorhebt, mit Ungenauigkeiten behaftet. Z. B. die vorgestellten Linien haben eine gewisse Breite, ebenso wie sie nicht farblos sind usw. Kant meint eine gewissermaßen begriffliche Konstruktion, etwa gemäß den Postulaten Euklids. Bei einer solchen haben wir es mit einer reinen Gedankenkonstruktion zu tun, deren Ausführung wir uns nur denken, für welche die mechanischen Mittel, wie Lineal und Zirkel*) garnicht in Betracht kommen, und darum besitzt sie die volle Schärfe des Begriffs. Auf solchen anschaulichen Konstruktionen beruht es, daß die Geometrie von den Definitionen, die einen Begriff innerhalb seiner Grenzen genau bestimmen, ausgehen kann, ferner daß der Geometer im Gedankenexperiment mit dem geistigen Besitz seiner geometrischen Vorstellungen frei schalten und diese zur Fortführung des Systems oder zur Auffindung ron Beweisen beliebig kombinieren kann. Besonders verdient hervorgehoben zu werden, daß bei den geometrischen Beweisen nicht lediglich logische Folgerungen gezogen werden, daß ihre Sätze nicht analytische Urteile sind. “**)

*) „Die konische Gestalt wird man ohne alle empirische Beihilfe, bloß nach dem Begriff anschauend machen können, aber die Farbe dieses Kegels wird in einer oder anderer Erfahrung zuvor gegeben sein müssen." (Krit. d. rein. Vern. S. 549.)

*) Dies werde an folgendem Beispiel erläutert (Vergl. E. Mach, Erkenntnis u. Irrtum, 2. Aufl. S. 305). Der Satz, daß ein Peripheriewinkel eines Kreises halb so groß als der aut demselben Bogen stehende Centriwinkel ist, beruht freilich logisch auf dem Satz vom Außenwinkel eines Dreiecks. Aber um ihn zu finden, muß man zunächst ein gleichschenkliges Dreieck konstruieren, dann um dessen Spitze mit einem Schenkel einen Kreis beschreiben, man hat ferner sein Augenmerk auf die Lage der Winkel in Bezug auf den Mittelpunkt zu richten u.s.f. - lauter geistige Tätigkeiten, die mindestens ebenso wichtig sind wie die Vollziehung der betr. Syllogismen, und die man wohl unter dem Namen der "anschaulichen Konstruktion" zusammenfassen kann. Bis zu diesem Punkt sehen wir daher Uebereinstimmung bei Kant und Mach. Auch ein prinzipieller Gegensatz zwischen Kant und Wellstein (a. a. O. S. 111 u. f.) scheint uns nicht zu bestehen.

Um die Wichtigkeit der anschaulichen Konstruktion für die Mathematik zu zeigen, sei an die Darstellung der komplexen Zahlen durch Gauß erinnert, oder an die Realisierung der nichteuklidischen Geometrien durch Wellstein. 
Wie die „anschauliche Konstruktion“ für die Definitionen und Demonstrationen von grundlegender Bedeutung ist, so beruht nun nach Kant auch auf ihr, daß die Geometrie „der Axiome fähig ist, weil sie vermittelst der Konstruktion der Begriffe in der Anschauung des Gegenstandes die Pıädikate desselben a priori und unmittelbar verknüpfen kann, z. B. daß 3 Punkte jederzeit in einer Ebene liegen."

Ist es nun „das Eigentïmliche allergeometrischen Erkenntnis, daß sie ihre Begriffe vorher in der reinen Anschauung darstellen müsse", so sieht Kant den Grund dafür darin. daß „die Geometrie eine reine Anschauung, nämlich die des Raumes, ihren Erkenntnissen zu Grunde legt"; - und damit kommen wir zur A priorität des Raumes. Alle räumlichen Bestimmungen, die wir an den Gegenständen unsrer Erfahrung wahrnehmen, "Ausdehnung, Ort, Gestalt, überhaupt der Raum mit allem, was ihm anhängig ist" sind nicht Prädikate äußerer Dinge, sondern sie werden von Kant, wie Farbe und Geschmack, zu „,bloßen Erscheinungen gezählt". Der Raum ist nicht etwas für sich Existierendes, auch keine Beziehung oder Ordnung zwischen realen Objekten, sondern die Form, in der wir unsre Empfindungen anordnen, unter der wir deren Mannigfaltigkeit zu einer Einheit zusammenfassen und objektivieren. Die Vorstellung des Raumes beruht nicht auf der Kombination von Wahrnehmungen endlicher Räume, sondern die äußere Erfahrung setzt selbst schon die Raumvorstellung voraus; diese ist die Bedingung aller Erfahrung.

Wir können hier auf die Begründung, die Kant seiner Lehre gegeben hat, nicht näher eingehen. Wir bemerken nur, daß es zum Verständnis seiner Auffassung auf Folgendes ankommt. Einmal ist der Bestandteil in unsern Wahrnehmungen, der die räumlich-zeitliche Bestimmtheit enthält, gegenüber den Sinnesqualitäten - Farben, Tönen, Wärmen usw. - das feste Gerüst, das beharrt, wenn jener Inhalt wechselt, oder der tatsächlich mit verschiedenem Inhalt erfüllt werden kann. Er ist das konstante Element, das alle unsre Wahrnehmungen begleitet. Wie ferner die Existenz der Geometrie zeigt, lassen sich aus wenigen Sätzen heraus, - welche die Art unsrer 
Raumanschauung festlegen - im voraus mögliche Raumerfahrungen bestimmen, während über das Auftreten einer bestimmmten Empfindungsqualität nur die Erfahrung belehren kann. Daher ist es eine unbestreitbare Tatsache, daf die räumlich-zeitliche Bestimmtheit unsrer WVahrnehmungen gewissermaßen einen höheren Erkenntniswert besitzt als die mehr zufällige und in weit größerem Maße subjektiven Täuschungen ausgesetzte Empfindungsqualität. Das war der erste Punkt, den es zu erklären galt. Sodann hatte Kant die Grundüberzeugung - und das steht nach ihm und durch ihn unbestreitbar fest - daß sich unsre Erkenntnis nur auf Erscheinungen bezieht, daß wir über das Wesen der Dinge an sich nichts wissen können. Dann kann aber dem Raum keine erkennbare Realität zukommen; auch kann er keine Bezielıung zwischen realen Objekten sein. So blieb, nach Kant, nichts übrig, als daß die Raumvorstellung eine Gesetzmäßigkeit unsers Bewußtseins ist, die Materie der Empfindungen $\mathrm{zu}$ ordnen. Dann ist sie in der Tat die Bedingung aller möglichen Erfahrung.*)

Ist nun die Raumanschauung die Form, das Gewand, in das wir alle Sinneseindrücke kleiden müssen, so sind die grundlegenden Sätze, in denen sie sich darlegt — die Axiome der Geometrie - allgemein und notwendig für jeden speziellen Erfahrungsinhalt. Unser Geist kann frei schöpferisch mit diesen ihm immanenten Formelementen verfalıren und doch für die empirische Wirklichkeit gültige Gesetze entwickeln. So erklärt es sich, daß die Geometrie ron der Erfahrung unabhängig ist und doch für diese objektive Gültigkeit besitzt. Das ist das Resultat, das Kant sehr wichtig war: die Mathematik ist kein bloßes Hirngespinst, sondern für die Naturerkenntnis von grundlegender Bedeutung. So konnte er sagen, daß in jeder besonderen Naturlehre nur

*) Daß die Raumvorstellung wie ein fertiges Schema uns eingeboren ist, oder daß sie zeitlich allen Wahrnehmungen voranginge, hat Kant nicht behauptet. Vielmehr ist mit seiner Ansicht wohl vereinbar, daß jene sich im Menschengeschlecht oder im einzelnen Individuum mit den Sinneserfahrungen entwickelt. 
soviel eigentliche Wissenschaft angetroffen werden könne, als darin Mathematik anzutreffen ist. Darnach galten ihm die nur beschreibenden Zweige der Naturforschung und diejenigen, die wie die Chemie, auf Erfahrung und Experiment beruhen, nicht für vollgültige Wissenschaften.*)

Das andere Resultat — das freilich in ganzer Sehärfe erst auf Grund der modernen Entwickelung der Geometrie (vergl. Abschn. 11) ausgesprochen werden konnte - ist, daß wir in allen unsern Anschauungen an den euklidischen Raum und seine Gesetzmäßigkeiten, wie sie in den Axiomen zum Ausdruck kommen, gebunden sind. So ist dieser Raum samt den euklidischen Axiomen eine Anschauungsnotwendigkeit, aus der dann die ganze euklidische Geometrie mit Notwendigkeit hervorgeht, und aus der sich ihre einzigartige Bedeutung erklärt. Abweichende Axiome - etwa das zu dem 5. Postulat Euklids im Gegensatz stehende, daß durch einen Punkt zu einer Geraden zwei Parallelen gezogen werden können mögen in sich keinen logischen Widerspruch enthalten, auch nicht mit andern Axiomen in Gegensatz treten, aber intuitiv sind sie nicht vorstellbar. Solche Annahmen mögen von mathematischem Interesse sein, aber für die Naturerkenntnis haben sie keine Bedeutung. - Gibt man endlich mit Kant den apriorischen Charakter unsrer gesamten Ranmanschauung zu, so daß sie in der Tat von der Erfahrung vollständig unabhängig ist, so erklärt sich auch das Evidenzgefühl, das wir den geometrischen Axiomen gegenüber haben, ${ }^{* * *}$ ) da wir

*) Dieser Mangel muß schließlich auch für jedes Gebiet, das sich aul "Tatsachen-Wissen * bezieht, gelten — d. h. auch für die Geschichtsforschung. Kein Wunder, daß sich bei der großartigen Entwicklung der Natur- und Geschichtsforschung der nachkantischen Zeit und der Durchbildung ihrer Methoden gegen jenen engen Begriff der Wissenschaft Widerspruch erhob, und aus dem Geist dieser Wissenschaften heraus eine Um- und Neubildung der Ideen Kants versucht wurde

**) So in den Schriften von O. Liebmann. (Vergl. E. Adickes „Liebmann als Erkenntnistheoretiker“, Festschrift d. „Kantstudien “1910.)

**) Den Einwendungen, die Adickes gegen Liebmann vom Standpunkt des Empirismus erhebt, pflichten wir bei. Aber der strenge 
uns bei ihnen in ähnlicler Lage befinden. wie gegenüber den logischen Axiomen. Dieses Gefühl muß sich bei uns einstellen, weil in unsrer geistigen Organisation der Zwang begründet liegt, so und nicht anders vorzustellen.

Soweit die Lehre Kants und ihre Konsequenzen. Fürwahr eine Auffassung von imponierender Geschlossenheit und doch findet sie bei Mathematikern, Naturforschern und Philosophen Widerspruch.

Einem Teil von ihnen ist Kant nicht rationalistisch genug. Der „sensualistische Erdenrest“ (Wellstein), der seiner "Anschauung" zu Grunde liegt, bildet den Stein des Anstoßes. Einem andern Teil, in Anlehnung an die biologische und experimentelle Naturforschung unsrer Zeit, ist Kant zu sehr Rationalist, ilım scheint es unmöglich, die "Totalität der Wirklichkeit in ein Netz von einigen wenigen klaren und deutlichen Verstandesbegriffen einzufangen“. Sie betont die empiristische Grundlage unsers gesamten Wissens - auch des mathematischen. Im einzelnen: sie bestreitet die Apriorität der Raumanschauung*) sowohl, als speziell der Geometrie, und damit die Notwendigkeit der euklidischen Axiome, sie bestreitet ferner, daß die Anschauung die grundlegende Erkenntnisquelle der Geometrie sei.**)

*) U. a. wird von psychologischer Seite hervorgehoben, daß unsre Raumvorstellungen nicht als bloße subjekive Formen unsers Anschauens gewonnen werden, sondern daß die Sinnesempfindungen sie mannigfach beeinflussen. Die absolute Scheidung des Inhalts unsrer Empfindungen von der Form der Raumanschauung, wie sie Kant vorgenommen hat, rechtfertigt sich also nicht. Vielleicht kann man sogar den Sinnesempfindungen die Raumempfindungen an die Seite stellen und von einer durch Erfahrung gewonnenen Raumanschauung sprechen. Somit ist es für die Erkenntnislehre durchaus begründet, wenn man sich auf den Standpunkt stellt, daß mit den Sinnesdaten wie die zeitliche, so die räumliche Bestimmtheit - d, h. Ort, Gestalt und Lage - ursprünglich gegeben ist. (Schuppe, Grundr. d. Erkenntnistheorie und Logik, S. $77 \mathrm{f}$.)

*) Eine Verteidigung der Kantischen Auffassung s. E. König, („Kant u. die Naturwissenschaft.")

Kantianer wird einen außerhalb des Denkens vorhandenen Erfahrungsraum (S. 31 a. a. O.) bestreiten. 
10. Kant hatte zwischen Rationalismus und Empirismus in der Art vermittelt, daß er der Erfahrung den Stoff, der Vernunft die Form der Erkenntnis zuwies. Kann sich der Empirismus mit dieser absoluten Sonderung der empirischen und apriorischen Elemente zufrieden geben? Nach Fr. Paulsen (Einleitung in die Philosophie) kann er es nicht. Wohl wendet sich Paulsen gegen die Auffassung, daß die Seele ursprünglich ein leeres Blatt ist, worauf die Dinge vermittelst der Sinne ihre Zeichen eintragen. Schon die bloße Empfindung - Licht, T'on, Geschmack - wird unsrer Erkenntnis nicht von außen eingedrückt, sondern von ihr, bei der Begegnung mit der Umgebung, hervorgebracht als etwas, das so überhaupt nur in ihr als eigenes Erzeugnis ist; und auch die allgemeinen Formen der simnlich-anschaulichen Welt sind vom Subjekt spontan hervorgebrachte Ordnungen, keineswegs aber der Abdruck eines an sich seienden leeren Raumes ode: leerer Zeit. Wir mögen der an sich seienden Wirklichkeit „intelligibele" Ordnungen zuschreiben, denen unsre Anschauungsformen irgendwie korrespondieren, aber diese Formen sind nicht Impressionen, sondern Schöpfungen des Subjekts. Noch sichtbarer gilt dies ron den Begriffen . . . .Wir werden also sagen: alle Erkenntnis ist Tätigkeit des Subjekts und als solche a priori. Freilich a priori nicht in dem Sinne, daß sie ein absolut beziehungsloser innerer Vorgang wäre; wie alle Betätigung, so ist auch die Betätigung der Intelligenz durch die Natur der Dinge, auf die sie sich richtet, mitbedingt." - In den letzten Worten tritt uns der Gegensatz; in dem Paulsen zu Kant steht, entgegen. Noch schärfer ist er in folgendem Satz formuliert: „Alle Naturgesetze, alle Wahrheiten von Tatsachen, auch die allgemeinsten, sind empirische Gesetze, freilich nicht in dem Sinne, daß die Natur draußen sie den Sinnen imprimiert, wohl aber in dem Sinne, daß der Verstand sie im Hinblick auf die in der Wahrnehmung gegebenen Zusammenhänge in Raum und Zeit ausbildet und ihre Wahrheit an ilınen kontrolliert." So wird die Erfahrung als Quelle aller unsrer Erkenntnis hingestellt und ihr auch die Kontrolle über ihre Richtigkeit zugewiesen; und es erklärt 
sich so, daß Paulsen unserm Tatsachenwissen den Charakter der Allgemeinheit abspricht und ihm nur präsumtive Allgemeinheit läßt, d. h., daß Berichtigung durch nachfolgende Erfahrung vorbehalten bleiben muß. In gleicher Weise wie für Hume sind ferner auch für Paulsen nur die Lehrsätze der reinen Geometrie von apodiktischer Geltung, weil sie sich auf Idealbegriffe beziehen. Jede Anwendung dieser Sätze ist empirischer Natur und enthält deshalb eine Berufung auf Erfahrung. - Kant hatte den alten rationalistischen Standpunkt nicht überwunden, als ob die Sinneswahrnehmung als die niedrigere der höheren Verstandeserkenntnis gegenübertrete. Auch hier knüpfen wir, um die allgemeine Art seines Wesens zu kennzeichnen, an ein Wort Paulsen's*) an. „Es ist die Platonisch-Aristotelische Grundanschauung, von der sich das Kantische Denken ïberall leiten läßt; der Gegensatz ron Vernunft und Sinnlichkeit gibt seiner ganzen Philosophie die Grundstruktur. So in der theoretischen Philosophie: Die Sinnlichkeit, die Empfindung, liefert bloß die Materie, der Verstand als gesetzgebendes Vermögen macht daraus erst Erkenntnis. Und ebenso wieder in der praktischen Philosophie: Die Sinnlichkeit, Begierden und Neigungen, gibt die Materie des Wollens, die Vernunft als gesetzgedendes Vermögen schaffit erst die sittliche Welt, ein einheitliches Reich der Freiheit, wie dort ein Reich der Natur. Hier wie dort sind die Vernunftbestimmungen an ihrer absoluten Allgemeinheit erkennbar, dort als Naturgesetze hier als Sittengesetze sich darstellend. Und hier wie dort ist das Allgemeine und Gesetzmäßige das spezifische Menschliche, daher das Höhere und Vornehmere."

Dem widerspricht, kann man sagen, der Wirklichkeitsund Tatsachensinn unsrer Zeit, der auf allen Gebieten des theoretischen und praktischen Wissens mit dem Studium der Erfahrungstatsachen anhebt und in mühsamer Einzelforschung die vorhandenen Gesetzmäßigkeiten festzustellen sucht. Es widerstrebt ihm, daß sich die obersten Naturgesetze — wenn

*) Die Kultur der Gegenwart, systematische Philosophie, S. 288. 
anders sie mehr sein wollen als formale Allgemeinheiten z. B. die Erhaltung der Substanz, als Gesetzmäßigkeiten des erkennenden Bewußtseins sollen deduzieren lassen, während doch die Geschichte der Wissenschaft zeigt, wie lange es gedauert hat, und wie mühsamer Forschung es bedurfte, solche Prinzipien zu gewinnen. Darum muß auch bei ihnen der Erfahrung ein wesentlicher Anteil gesichert werden.

Dieser Gegensatz zu Kant erstreckt sich auch auf die Geometrie. "Geometry is a physical science". Dieses Wort des englischen Mathematikers und Philosophen Clifford bezeichnet kurz die Ansicht der meisten neueren Mathematiker und Naturforscher, die in ihrem Ursprung auf Gauß zurïckgeht.*)

11. Den unmittelbaren Anlaß zu dieser Aenderung der Anschauungen gab die Aufstellung der nichteuklidischen Genmetrien, d. h. solcher geometrischen Systeme, die von anderen Voraussetzungen ausgehen, als sie dem euklidischen Lehrgebäude zu lirunde liegen. Die Anregung hierzu ist von K. F. Gauß ausgegangen, und von ihm sind früher als von andern Forschern wesentliche Ergebnisse gefunden worden, die er freilich im Zusammenhang nicht veröffentlicht hat. Er hat ferner den prinzipiellen Gegensatz, in den ilın seine Entdeckungen zu Kant brachten, klar erkannt und ausgesprochen. Fast gleichzeitig mit Gauß haben der Russe Lobatschefskij und der Ungar J. Bolyai ihre Ideen entwickelt, in dem Ausbau derselben sicherlich unal,hängig von Gauß.**)

*) Zahl und Raum nehmen keine Sonderstellung gegenüber ailen übrigen Erkenntnisgegenständen ein. Die ihnen zugewandten Denkformen genießen den Vorzug der mathematischen Evidenz und mathematischen Gewißheit nicht als ausschließliches Vorrecht. Die Mathematik nimmt im System der Wissenschaft nicht eine isolierte, in keiner Einteilung der anderen Wissenschaften unterzubringende Stellung ein." (A. Höfler, Didakt, d. math. Unterr. S. 454.)

**) Betreffs der Vorgeschichte der nichteuklidischen Geometrien mub auf die Spezialliteratur hingewiesen werden. Auch ist es nicht möglich, von ihrem Inhalt mehr als geringe Proben zu geben. Für uns handelt es sich hier zunächst hauptsächlich um die Grundgedanken, die in der 1. Periode ihrer Entwicklung zum Ausdruck kommen. S. F. Klein (Vorlesungen über nichteuklidische Geometrie). 
Wir hatten schon früher (S. 11) hervorgehoben, daß sich unter den Grundvoraussetzungen Euklids das 5. Postulat nach Form und Inhalt, von den übrigen unterscheidet. Auch betreffs seiner Anwendung im Lehrsystem nimmt es eine Ausnahmestellung ein. Während jene-bereits bei den ersten Beweisen zur Geltung kommen, wird es erst bei dem 29. Lehrsatz des 1. Buches angewandt. Die Sätze 1 bis 26, welche 11. a. von der Kongruenz der Dreiecke, den Beziehungen Zwwischen ihren Seiten und Winkeln, von Neben- und Scheitelwinkeln, sowie von einigen Eigenschaften gleichschenkliger Dreiecke handeln, können ohne jenes Parallelen-Axiom bewiesen werden, sind also von ihm unabhängig. Anch bei den Sätzen 27 und 28 (zwei Gerade sind parallel, wenn die Wechselwinkel gleich sind oder die Gegenwinkel usw.) gelingt der Beweis ohne das 5. Postulat. Nur bei der Umkehrung, daß nämlich bei parallelen Geraden jene Winkelpaare gleich oder supplementär sind, wird es angewandt. An einer späteren Stelle des Systems wird es dann nie wieder erwälnt. "Euklid hat demnach erkannt, daß ein Satz, der für sein Lehrgebäude von hervorragender Wichtigkeit ist, mit Hülfe der übrigen Voraussetzungen nicht bewiesen werden kann. Er verschmäht es, einen Scheinbeweis zu liefern; vielmehr sucht er (so müssen wir aus seiner Behandlungsart schließen) dem Leser das Vorhandensein einer Lücke zum vollen Bewußtsein zu bringen." (Killing-Hovestadt a. a. O. S. 26). Und diese Lücke zu schliefen, haben die Bemühungen zahlloser Mathematiker, von denen die ersten gegen 2000 Jahre zurückliegen, nicht vermocht. Alle die vorgebrachten Beweise haben genauer Prïfung nicht Stand halten können, da in ihnen stets irgend ein dem euklidischen äquivalentes Axiom versteckt angewandt war.*) - Ja diese Versuche mußten scheitern, wie

*) In den meisten Lehrbüchern wird es ersetzt durch das Axiom "durch einen Punkt kann man zu einer Geraden nur eine Parallele ziehen." Von derartigen Axiomen sagt G. S. Klügel (1763), der Verfasser einer Kritik der berühmtesten Versuche, das 5. Postulat zu beweisen: "Wir wissen es nicht infolge strenger Schlüsse oder 
von der Wissenschaft jetzt einwandfrei festgestellt ist. „Das Par.-Ax. ist von den übrigen Axiomen unabhängig, keine mathematische Folge aus ihnen. Man kann sogar ein konsequentes Lehrgebäude auf Grund der übrigen Axiome aufbauen, welches das Lehrgebäude der euklidischen Geometrie nur als einen speziellen Teil umfaßst." (F. Klein, mathemat. Annalen Vl S: 113). - Der erste, der ein derartiges Lehrgebäude ohne das Par.-Ax. auf Grund der 28 ersten Propositionen Euklids aufstellte, war eben Lobatschefskij. Wir greifen auf schon Gesagtes zurïck. Wenn eine Gerade zwei andere unter gleichen Gegenwinkeln schneidet, so sind die beiden Geraden parallel. Denn sonst müßten sie, wie sich leicht zeigen läßt, 2 Punkte gemeinsam haben; sie müßten zusammenfallen. (Vergl. Baltzer, d. Elem. d. Matlı. II. Bd., VI. Aufl. S. 12). Läßt man nun in einem Dreieck ABC, in dem CD senkrecht zu AB gezogen ist, AB wachsen, (wobei der Punkt A festgehalten wird) so nimmt der Winkel ACB zu. Gibt es nun einen Grenzwert, dem dieser Winkel zustrebt, wenn $B$ immer weiter auf AB fortrückt, oder wenn AB immer mehr wächst?

Die uns geläufige Anschauung der Figuren wird unbedenklich antworten: Dieser Grenzwinkel wird erreicht und nur dann erreicht, wenn Winkel ACB den Winkel ABC zu $2 \mathrm{R}$ ergänzt, und sie wird diesen Grenzwinkel - darin besteht seine Wichtigkeit - zur Konstruktion der „Parallelen“ zu AB benutzen. Aber mit jener Aussage über die Größe des Grenzwinkels überschreiten wir unsre Erfahrung. Denn diese kann sich nur auf endliche, nicht auf unendliche Raumerstreckungen beziehen. Auch überschreiten wir das Gebiet logischer Deduktion. Denn einen Beweis kann man, wie dargelegt wurde, für die Richtigkeit der angenommenen Größe des Grenzwinkels nicht erbringen. Wir machen daher mit der Festsetzung des euklidischen oder eines äquivalenten Axioms eine Annahme, der ein gewisses willkürliches Moment

vermöge deutlicher Begriffe von der geraden und der krummen Linie, vielmehr durch die Erfahrung nnd durch das Urteil unsrer Augen" ein Satz, dessen positiver Teil freilich zu beanstanden ist. 
anhaftet - eine Substruktion, eine „Unterführung“ (A. Höfler) unsrer Raumerfahrungen, die uns instand setzt, mit genauen Begriffsbestimmungen $\mathrm{zu}$ arbeiten. Unsre gesamte Erfahrung stebt mit dieser Annahme nicht in Widerspruch. A ber sie zwingt uns nicht zu jener. Tatsächlich gehen wir also schon mit der euklidischen Geometrie über unsre Erfahrung hinaus. Die Erfahrung beweist weder die Richtigkeit des angenommenen Axioms. noch kann sie es widerlegen. Man kann nur sagen, daß sie mit der euklidischen Annahme nicht in Widerspruch steht, daß ferner letztere für die begriffliche Darstellung der Tatsachen des Naturgeschehens die einfachste ist, und daf sie unserm Vorstellungsvermögen am nächsten kommt. - So erklärt es sich, daß auch noch andere Annahmen versucht worden sind; und das haben Gauß, Lobatschefskij und Bolyai getan, indem sie annahmen, daß der oben erwähnte Grenzwinkel schon etwas früher erreicht wird, bevor der bewegliche Winkelschenkel mit der euklidischen Parallele zusammenfällt - denn überschreiten kann er sie nach dem vorausgegangenen Lehrsatz nicht. Dann verlangt aber die Symmetrie, daß es aut der andern Seite von CD eine zweite „Parallele" gibt, die mit $C D$ den gleichen Winkel bildet, wie die erste. Ja, verlängern wir diese Parallelen über ihren Schnittpunkt linaus, so liegt in 2 der so gebildeten Winkelfelder ein ganzes Strahlenbüschel von Geraden, die AB nicht schneiden. Diese Annahme, daß man durch C 2 Parallelen zu AB ziehen kann, die damit übereinstimmt, daß eine Gerade AB 2 unendlich ferne (reelle) Punkte hat - während diese bei Euklid zusammenfallen - oder daß die Winkelsumme eines Dreiecks kleiner ist als $2 \mathrm{R}$, hat nun Lobatschefskij seiner Geometrie anstatt des 5. Postulats zu Grunde gelegt, während er die übrigen euklidischen Axiome beibehält. Sein Hauptverdienst ist aber, daß er für diese Geometrie ein vollständiges System trigonometrischer Formeln aufgestellt hat, durch die er " die Länge der gekrümmten Linien, den Flächeninhalt der Oberflächen und den Rauminhalt der Körper berecinnen kann." Er hat also in der Tat gezeigt, caß ein in sich widerspruchloses geometrisches Lehrgebäude ohne das 5. Postulat möglich ist. Ja noch mehr, unsre 
menschliche Erfahrung, die gegenüber der Unermeßlichkeit des Weltenraumes nur ein unendlich kleines Raumgebiet umfaßt, steht mit seiner Geometrie nicht in Widerspruch. Denn die Formeln Lobatschefskij's enthalten eine gewisse Konstante, und wenn diese unendlich groß genommen wird, so ergeben sich die gewöhnlichen trigonometrischen Formeln, und das heißt soviel, daß jene Formeln für ein unendlich kleines Raumgebiet mit der euklidischen Geometrie in Einklang stehen.

Aber es gibtaußer der Geometrie Euklids - der parabolischen - und derjenigen von Lobatschefskij — der hyperbolischen noch eine dritte gleichberechtigte - die elliptische -, deren Begrïndung auf B. Riemann zurückgeht. Der geläufigen Anschanung erstreckt sich die gerade Linie nach entgegengesetzten Richtungen ins Unendliche. Das ist, sagt der Empirismus, wieder ein Urteil, das auf einer Annahme beruht, die unsre Erfahrung äberschreitet. Jedenfalls lassen sich unsre tatsächlichen Raumerfahrungen mit der Auffassung in Einklang bringen, daß die Gerade eine in sich geschlossene Linie ist*) - eine Auffassung, durch welche der Raum endlich wird, (wenn er auch unbeg enzt bleibt), und in sich zurückkehrt; während die Unendlichkeit der Geraden auch die Unendlichkeit des Raumes postuliert.

Kehren wir nun noch einmal zu dem oben betrachteten Grenzwinkel zurück. Ist AB eine geschlossene Linie, so gelangt der Punkt B, wenn er weiter und weiter fortrückt, schließlich nach $A$. CB löst sich also nie von AB ab, es gibt dann keinen Grenzwinkel und damit auch keine Parallele zu AB. In der auf dieser Annahme aufgebauten Geometrie beträgt die Winkelsumme eines Dreiecks mehr als $2 \mathrm{R}$ analog der Grundeigenschaft eines sphärischen Dreiecks aber der Ueberschuß über $2 \mathrm{R}$ ist um so kleiner, je kleiner die Fläche des Dreiecks ist. In gleicher Weise hängt in der

*) Um ein Beispiel anzuführen, das sich aber nicht mit dem Folgenden deckt, sei an einen Kreis mit großem Radius erinnert. Wird dieser groß genug gewählt, so kann man es dahin bringen, daß die Abweichung einer Sehne von dem zugehörigen Kreisbogen unterhalb der Beobachtungsfehler liegt. 
hyperbolischen Geometrie der Ueberschuß von $2 \mathrm{R}$ über die Winkelsumme des Dreiecks von dessen Flächengröße ab. Für ein unendlich kleines Dreieck wird der Ueberschuß in beiden Fällen gleich Null. Somit fallen im Unendlichkleinen alle drei Geometrien zusammen, und menschliche Erfahrung kann schon aus diesem Grunde keine von ihnen für „richtiger“ erklären, als die andere. Es ergeben sich also aus der Parallelentheorie im drei-dimensionalen Raum drei gleichberechtigte Geometrien. Jeder von ihnen eignet ein bestimmter Raumbegriff, der durch die zugrundegelegten Axiome bestimmt wird.*)

Indem wir die genauere Darlegung dieser Ergebnisse und ihrer Konseqüenzen für spätere Ausführungen zurückstellen müssen, bemerken wir nur folgendes. Mit diesen Ergebnissen war in das scheinbar so feste Gefüge der euklidischen Geometrie Bresche gelegt. Es war gezeigt, daß nicht sie allein als geometrische Wissenschaft möglich sei, ja daß noch andere Geometrien zu einer begrifflich genauen Darstellung unsrer Raumerfahrungen dienen können, wenn uns auch die Vorstellbarkeit ihrer Raumformen - was freilich durchaus nicht unwesentlich ist - nicht gelingen will.

So verlor die euklidische Geometrie insofern ihren apriorischen, absoluten Charakter, als sie nicht als die Ausprägung einer subjektiven, aller Erfahrung zugrundeliegenden Raumanschauung zu betrachten ist; sie normiert vielmehr, durch Erfahrung veranlaßt, in ihren Axiomen einen bestimmten Raumbegriff, und die Erfahrung hat wieder darüber zu entscheiden, wie weit jene und damit die aus ihnen gezogenen Folgerungen auf das Naturgeschehen angewandt werden können. Dadurch trat die Geometrie in gewisser Weise in die Reilhe der Naturwissenschaften.**) Das ist eine Auffassung, die von S. 492 .

*) Für die euklidische Geometrie vergl. Wundt, Logik I. Bd.

$\left.{ }^{* *}\right)$ Die Durchführung derartiger Gedankengänge findet man in den Schriften von E. Mach, besonders in dem oben citierten Werk "Erkenntnis und Irrtum", sowie bei H. Poincaré „Wissenschaft und Hypothese"; eine kritische Beurteilung derselben bei W. Wundt, Logik I u. II. 
bedeutenden Mathematikern, so von Gauß, Graßmann, Clifford, direkt ausgesprochen wurde.

Dies heißt aber nicht, daß die Geometrie sich, wie die Naturwissenschaft, mit physischen Objekten beschäftigt, noch daß sie ihre Grundsätze oder die aus ihnen abgeleiteten Sätze als „experimentelle Tatsachen “ der Erfahrung entnehmen kann.*) Vielmehr bleibt es dabei, daß ihre Objekte in der absoluten Präcision, die in den Definitionen gefordert wird und in den Axiomen die Beziehungen der Raumgebilde regelt, eine rein ideale Existenz haben. Wohl liegen der Aufstellung der Definitionen und Axiome Beobachtungen an physischen Körpern zu Grunde, -.. und schon in dieser Hinsicht nimmt die Geometrie gegenüber der Naturwissenschaft keine Sonderstellung ein - das Wesen der geometrischen Grundsätze liegt jedoch, wie F. Klein mit Recht herrorhebt, in der I d e a l is ierung der empirischen Daten. Indem nun diese Daten, das ursprünglich Gegebene, verschiedene begriffliche Darstellungen oder Nachbildungen zulassen, kommt in die Auswahl der möglichen Axiomsysteme eine gewisse Wilikür, die allerdings dadurch beschränkt ist, daß dieses System mit den Tatsachen übereinstimmen muß und in sich keine logischen Widersprüche enthalten darf. Der Naturforscher, der in der Geometrie nu: ein Mittel zur Untersuchung des physisch Gegebenen sieht (E. Mach), wird dasjenige System auswählen, das die einfachste Darstellung der empirischen Daten zuläßt, ähnlich wie Zweckmäßigkeitsgründe dafür entscheidend sind, ob man das metrische oder ein anderes Maßsystem anwenden will.

So ist nun die Raumlehre nicht mehr, wie Kant es wollte, eine Bedingung für das Zustandekommen der Erfahrung, sondern ein Mittel ihrer begrifflichen Beherrschung. - Und auch die oben erwähnte Idealisierung soll keinen Gegensatz zu dem Verfahren der Naturforschung enthalten. Denn auch zu den Grundannahmen jeder Naturwissenschaft leite die

*) Vergl. Poincaré a. a. O. S. 51; F. Kilein, Vorles, übet nichteuklidische Geom. I, S. 354 . 
Erfahrung hin, und sie seien, ebenso wie die Grundannahmen der Geometrie, Idealisierungen dieser Erfahrung; *) und in demselben Sinne sagt Höfler ***) „Ebenso wie der exakte Forscher die Wirklichkeit dureh seine Definitionen und Annahmen unterfährt" - wenn er z. B. von vollkommen elastischen Körpern, idealen Flüssigkeiten und Gasen spricht -, „so unterfahren wir unsre räumlichen Anschauungen und Erfahrungen durch die Begriffe Punkt, Abstand, Richtung, Gleich u. s. f., aus denen sich die geometrischen Definitionen zusammensetzen."

Eine kritische Untersuchung dieser Ansichten müßte sich besonder's mit zwei Punkten befassen. Einmal wäre der Begriff "Erfahrung“ zu diskutieren, und andererseits wäre zu fragen, ob das Idealisierungsierfahren der Geometrie nicht doch wesentliche Unterschiede von dem in der Naturforschung geübten aufweist.

*) E. Mach a. a. O. S. 391.

**) Didakt. d. math. Unterr. S. 454. 
Erfahrung hin, der Geometrie. demselben Sin Forscher die Annahmen un elastischen Kö! „so unterfahı Erfahrungen Gleich u. s. f. zusammensetze Eine kri besonders mit "Erfahrung" ? ob das Ideal wesentliche L geübten aufwe

*) E. Mac

**) Didak

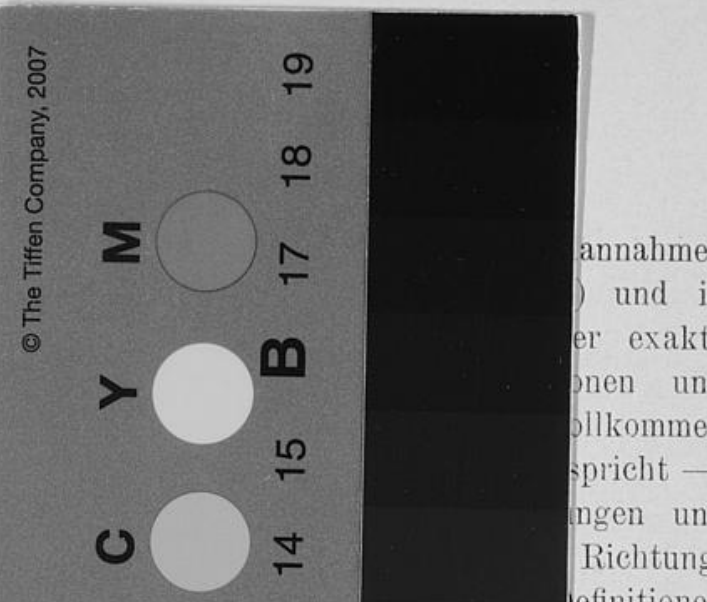

efinitionen

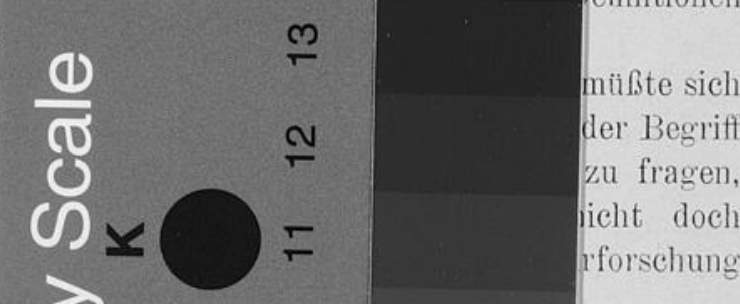

müßte sich der Begrift zu fragen, doch
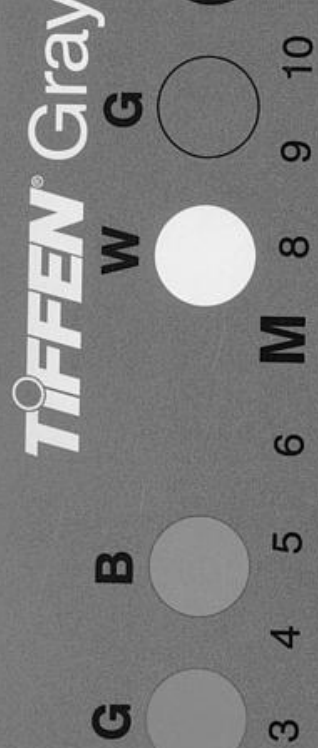

m

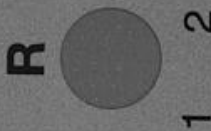

$\curvearrowright$

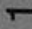



